\title{
Apprentissage du langage oral à l'école maternelle
}

Mise en œuvre d'un modèle polylogal d'apprentissage du langage oral en moyenne et grande section de maternelle en 2010-2013

Learning Oral Language in Kindergarten. Implementation of a Polylogical Model

of Oral Language Learning in Medium and Large Kindergarten in 2010-2013

\section{Pierre Péroz}

\section{(2) OpenEdition Journals}

Édition électronique

URL : https://journals.openedition.org/pratiques/3100

DOI : $10.4000 /$ pratiques.3100

ISSN : 2425-2042

Éditeur

Centre de recherche sur les médiations (CREM)

\section{Référence électronique}

Pierre Péroz, « Apprentissage du langage oral à l'école maternelle», Pratiques [En ligne], 169-170| 2016, mis en ligne le 30 juin 2016, consulté le 21 septembre 2021. URL : http://

journals.openedition.org/pratiques/3100; DOI : https://doi.org/10.4000/pratiques.3100

Ce document a été généré automatiquement le 21 septembre 2021.

(c) Tous droits réservés 


\section{Apprentissage du langage oral à l'école maternelle}

Mise en œuvre d'un modèle polylogal d'apprentissage du langage oral en moyenne et grande section de maternelle en 2010-2013

Learning Oral Language in Kindergarten. Implementation of a Polylogical Model

of Oral Language Learning in Medium and Large Kindergarten in 2010-2013

Pierre Péroz

1 L'apprentissage du langage oral à l'école maternelle fait l'objet d'observations convergentes de la part des chercheurs comme de celle des personnels d'inspection ${ }^{1}$ (MEN, 1999, 2011) depuis de nombreuses années ${ }^{2}$. Tous pointent la contradiction entre les objectifs affichés aux séances dites de langage et une parole magistrale qui occupe plus de la moitié de l'espace conversationnel. Dans le même temps, les acteurs du terrain posent tout aussi régulièrement les mêmes questions : comment gérer l'hétérogénéité des élèves souvent importante en langage, comment faire pour maintenir l'attention des élèves et comment évaluer les apprentissages des élèves.

2 C'est pour comprendre ce double constat d'une pratique perçue et vécue comme peu efficace que s'est constitué le groupe CLEA $^{3}$ composé d'enseignants-chercheurs en sciences du langage de l'Université de Lorraine et d'enseignants maitres-formateurs de Moselle, titulaires de diplômes de troisième cycle, doctorat ou DEA. Notre travail a d'abord été de mettre en évidence le caractère systémique de ces dysfonctionnements. Tout concourt en effet au développement de la parole magistrale au cours des séances de langage. Le modèle linguistique d'abord, dont le caractère scripto-centré (Lahire, 1993) appelle un retour régulier des reformulations magistrales à visée corrective ${ }^{4}$. Le modèle littéraire ensuite qui fait choisir comme support de séances des albums dont texte et images fonctionnent parfois de manière complexe, pénalisant d'autant les élèves les plus éloignés de la culture littéraire (Brigaudiot, 2000 ; Quet, 2009, Boiron \& Rebière, 2009). Enfin un modèle d'apprentissage « dialogal adulto-centré » (Péroz, 2015a) qui repose sur l'idée que comme dans la famille, «l'enfant apprend à parler avec l'adulte » a pour 
conséquence de privilégier l'individualisation des échanges mais aussi l'accélération du dialogue maitre - élèves pour, pense-t-on, permettre à chacun de s'exprimer ${ }^{5}$. Dans ce dispositif qui favorise par définition les échanges rapides, l'hétérogénéité du groupe est vite ressentie comme une gêne (Bautier, 2008). Les élèves les moins rapides ou les moins cultivés sont vite exclus de cette dynamique qui de plus, pose rapidement des problèmes de gestion, selon nos propres observations (Péroz, 2010). Trente pour cent des élèves ne prennent pas la parole et la longueur moyenne des interventions des autres élèves est de 6 à 8 mots (Florin, 1991), bien loin de ce qui serait nécessaire pour construire les « phrases complexes » ou acquérir les compétences discursives " raconter, décrire, expliquer etc.» attendues en grande section par les Programmes (2015) pour l'école maternelle.

Pourtant, nombre de travaux suggèrent qu'un autre modèle d'apprentissage est possible. Il y a d'abord les courants des pédagogies actives qui ont donné quelques preuves de l'intérêt des apprentissages coopératifs. Ce sont aussi des recherches sur l'acquisition de la part d'auteurs comme F. Winnykamen (1990) qui ont montré l'importance de "l'imitation $»^{6}$ dans tous les apprentissages. Dans l'apprentissage du langage en particulier, la description de F. François (1993) des enchainements par «continuité et mouvements » d'un élève à l'autre, a permis de voir comment dans un contexte favorable, c'est-à-dire d'écoute, les élèves apprenaient ensemble à parler. Dans le domaine de la didactique de l'oral, É. Nonnon (1997) a fait la critique du «dialogue pédagogique ordinaire » dont nous disions plus haut les conséquences négatives dans le contexte de séances de langage à l'école maternelle. En s'appuyant elle-même sur les travaux de F. François et d'A. Culioli, elle souligne le caractère essentiel du travail énonciatif, la nécessité pour tous les élèves de pouvoir reprendre, déplacer, reformuler, non seulement ce qu'ils entendent mais aussi ce qu'ils ont déjà dit.

4 Tirant les conséquences de ces observations critiques, nous faisons l'hypothèse que la « reprise-reformulation » (François, 1993) - par les élèves eux-mêmes - est au cœur de l'apprentissage du langage oral. Notre objectif de recherche est de définir les conditions favorables au développement des formes d'apprentissage collaboratif, et donc de développer et valider un modèle " polylogal » (Péroz, 2015a) d'apprentissage du langage oral à l'école maternelle, fondé sur les « reprises-reformulations » entre élèves à partir du texte support.

5 Nous présenterons ici le dispositif mis en place et les résultats obtenus en les comparant avec ceux que l'on peut trouver dans la littérature existante.

\section{Dispositif}

6 La recherche CLEA est une "recherche pragmatique » au sens où l'entend J.-P. Astolfi (1993), à la fois théorique et pratique. Théorique, parce qu'elle vise la construction d'un modèle théorique cohérent, le modèle « polylogal », différent du modèle d'apprentissage qui a fait l'objet des critiques que nous avons citées. Pratique, parce qu'elle vise à le valider dans les conditions habituelles de classe à l'école maternelle. Mais sur quels critères? C'est d'abord la participation des élèves. Les chiffres cités plus haut, les critiques adressées aux séances menées dans le cadre du dialogue pédagogique ordinaire, comme les questions récurrentes de la part des enseignants de terrain confirment l'importance de ce critère, apparemment trivial. 
7 Aussi, une approche quantifiée de la participation des élèves a-t-elle été retenue comme un premier outil d'évaluation dans le cadre du projet CLEA. On verra qu'indépendamment de sa simplicité elle est à l'origine d'un questionnement ouvert sur l'ensemble de la séance, dimension linguistique comprise.

\subsection{Recueil des données}

8 Le projet a combiné le travail d'une équipe pluricatégorielle d'une dizaine de chercheurs et celui d'une équipe d'une quinzaine d'enseignants volontaires en grande et moyenne sections de septembre 2010 à juin $2013^{7}$. Au total, 24 enseignants différents ont participé au projet. Certains sont restés d'une année sur l'autre, tandis que d'autres ont pu rejoindre le groupe la deuxième année alors que quelques-uns ont pu le quitter parce qu'ils changeaient de poste. Depuis la fin 2013, le groupe de recherche se consacre à l'exploitation des données recueillies en vue de la publication des résultats.

Les enseignants se trouvaient dans deux circonscriptions différentes de Moselle et plus d'un tiers de leurs classes se trouvait dans des zones sensibles ou des dispositifs qui ont succédé aux ZEP.

Au total, 78 séances de langage ont été enregistrées, 44 en grande section et 34 en moyenne section. Pendant les deux premières années, une séance a été enregistrée par chaque enseignant à la fin de chaque semestre. Au cours de la troisième année, toutes les séances ont été enregistrées au deuxième semestre par les enseignants qui avaient souhaité poursuivre le projet. Au total, les nombres sont assez équilibrés.

Tableau 1. Nombre de classes concernées et de séances enregistrées au cours du projet CLEA

\begin{tabular}{|l|l|l|l|l|l|l|}
\hline & \multicolumn{2}{|l|}{$2010-2011$} & \multicolumn{2}{|l|}{$2011-2012$} & $2012-2013$ & \\
\hline Semestres & 1 & 2 & 1 & 2 & 2 & \\
\hline Nombre de classes concernées & 12 & 15 & 15 & 13 & 7 & \\
\hline Nombre de séances enregistrées & 12 & 18 & 16 & 17 & 15 & 78 \\
\hline
\end{tabular}

11 Chaque année, plus d'une centaine d'élèves différents, répartis entre les différentes classes associées au projet, ont participé à ces séances de langage. Nous proposions aux enseignants de travailler avec des groupes d'élèves compris entre 6 et 15 élèves. Ce sont donc ces nombres que l'on retrouve dans les groupes, en MS, les groupes comptent de 5 à 13 élèves avec une moyenne de 9 élèves et en GS les groupes comptent de 6 à 16 élèves avec une moyenne à peine supérieure de 10 élèves.

12 La transcription de ces séances a permis la constitution progressive d'un corpus d'environ 140000 mots ou 14000 lignes dans un fichier Excel ${ }^{8}$.

\subsection{Articulation formation/recherche}

Ce résultat a été obtenu grâce à une collaboration approfondie entre les deux équipes qui se retrouvent trois fois par an pour des sessions de formation et d'échanges de trois 
heures, prises pour les enseignants de terrain sur leur temps de formation continue grâce au soutien des inspecteurs des circonscriptions concernées?.

La session de début d'année a pour but de former les enseignants à la mise en œuvre du modèle polylogal d'apprentissage du langage oral. Les deux autres sessions, à la fin de chaque semestre, sont consacrées au suivi de l'équipe et à l'approfondissement de la formation. Chaque séance de langage dont la transcription a été transmise auparavant au groupe de recherche fait l'objet d'une analyse qui est présentée à l'ensemble des participants qui en discutent ensuite les éléments. Ainsi, la dimension contractuelle du projet, structure et facilite les échanges entre les deux équipes. Si d'un côté, les enseignants prennent le risque de modifier leur pratique et qui plus est de la soumettre au regard des chercheurs par le biais des transcriptions de leurs séances, d'un autre côté, ils profitent en retour d'une analyse approfondie de leur travail : à l'issue de la session, chaque enseignant reçoit une version écrite de l'analyse de sa séance et éventuellement de celles des autres s'il le souhaite. Malgré les remises en cause que ces analyses peuvent générer, elles sont toujours jugées très positivement. Pour les enseignants de terrain, ce retour sur la pratique débouche sur une meilleure compréhension des phénomènes interactionnels (reprises-reformulations entre élèves) et une appréhension plus juste du travail énonciatif accompli par les élèves lors de leurs interventions (allongement, complexification des énoncés). C'est donc l'ensemble des séances conduites au cours de l'année par l'enseignant qui profitent de ce dispositif, même si nous n'aurons accès qu'à deux séances par an enregistrées et transcrites à fin d'analyse, au milieu de chaque semestre. Pour les membres du groupe de recherche, les moments de formation sont l'occasion de mesurer la pertinence du travail effectué en amont. Mais c'est aussi l'occasion d'approfondir des concepts propres au protocole de recherche. C'est ainsi que la notion de «format de séance $»^{10}$ a largement bénéficié du questionnement des enseignants au cours de ces rencontres.

Une série d'entretiens semi-directifs conduits à l'issue de la deuxième année avec la majorité d'entre eux a montré l'intérêt de ce suivi au long cours des enseignants qui soulignent tous l'efficacité de ce compagnonnage scientifique. Ils disent aussi la difficulté essentielle qu'a représentée le changement de posture pédagogique qui leur était demandé, bien plus délicat par exemple que l'abandon des supports illustrés à partir de la deuxième année ${ }^{11}$. En s'engageant dans le projet en effet, les enseignants acceptaient de modifier leur pratique selon des principes que nous allons maintenant évoquer.

\subsection{Un cadre pour les séances de langage}

Depuis une vingtaine d'années, tous les textes de référence ${ }^{12}$ pour l'école maternelle élargissent l'apprentissage du langage oral à tous les domaines d'activités qui sont, naturellement, l'occasion d'interactions langagières variées avec les élèves. Il est clair qu'il existe bien des situations différentes au cours desquelles les élèves apprennent à parler à l'école. Mais avec la même constance, les Programmes affirment l'importance de séances spécifiques prenant comme support des ouvrages de la littérature de jeunesse : écoute et rappel de récits en particulier. Les enseignants conçoivent d'ailleurs les choses de la même manière. C'est donc pour nous inscrire dans une pratique commune aux enseignants ${ }^{13}$ que nous leur proposons de travailler dans le cadre de séances de langage sur des supports littéraires. 
17 Mais la séance de langage sur album, véritable "genre scolaire » selon M. Jaubert et M. Rebière (2011) pose par ses caractéristiques mêmes des difficultés identifiées de longue date et que nous avons déjà partiellement évoquées.

La nature incertaine de la communication entre les participants fait de la séance un moment unique au déroulement parfois imprévisible tant pour l'enseignant que pour l'élève qui ne peut guère anticiper les questions qui vont lui être posées (Perrenoud, 1991).

19 Le caractère labile du langage conduit à une évaluation immédiate de chaque intervention qui conduit elle-même à une accélération des échanges dont on connait les effets négatifs sur la participation des élèves (Florin, 1995).

Enfin, la dimension didactique de la séance se traduit d'abord par le choix d'un support qui est presque toujours un album de la littérature de jeunesse. Malgré les qualités du genre qu'il n'est pas utile d'énumérer ici, plusieurs voix et non des moindres comme F. Grossmann (1996b) ou M. Brigaudiot (2000) ont souligné les problèmes énonciatifs que posent aux élèves la coprésence des deux modes narratifs iconique et textuel.

21 C'est donc pour répondre à ces difficultés que nous avons proposé aux enseignants d'adopter pour toutes les séances de langage un cadre commun, qui se décline sur trois plans : le plan des apprentissages, les plans didactique et pédagogique.

\subsubsection{Le plan des apprentissages}

22 Le plan des apprentissages correspond à la troisième relation construite dans le triangle didactique (Halté, 1992). Il caractérise dans notre dispositif les choix faits par l'enseignant pour favoriser la clarté cognitive relativement à l'apprentissage visé. Il s'agit en particulier pour les enseignants d'adopter à chaque séance un même "format ", c'est-àdire une même structure de questionnement dont la régularité, à l'instar $d u$ "format » interactionnel brunérien, favorise l'anticipation des élèves relativement aux questions qui vont lui être posées et leur compréhension de ce qui se joue dans la séance de langage. Suivant en cela les analyses d'É. Bautier et R. Goigoux (2004), nous avons pu constater que la régularité et la clarté du questionnement est un facteur d'intégration des enfants des milieux populaires qui composent plus d'un tiers des classes participant au projet. Deux questions essentielles structurent la séance. La première: «De quoi vous rappelezvous?", favorise une restitution aussi complète que possible mais pas nécessairement chronologique des événements. Le choix de cette première question repose sur l'idée, déjà développée par J. Giasson (1996), que la mémorisation des éléments du récit est à la base de sa compréhension. Cette question qui sera répétée plusieurs fois au cours de la première partie de la séance est clairement distinguée d'une consigne comme « racontez ce qui s'est passé » ou de toute autre consigne visant à la remise en ordre des événements. Ainsi, en accord avec les observations récentes de R. Goigoux et S. Cèbe (2012), nous considérons la chronologie comme un objectif qui n'a pas lieu d'être visé avant plusieurs séances sur la même histoire ${ }^{14}$. La seconde question qui vise, elle, à la compréhension de l'histoire, porte sur l'identité et les motivations des personnages. On sait le rôle structurant des personnages dans les récits de fiction pour la jeunesse (Reuter, 1988). K. Karmiloff et A. Karmiloff-Smith, (2003), ou plus récemment M.-F. Bishop et P. Joole (2012), ont souligné l'importance de l'identification des états mentaux et des motivations des personnages dans la compréhension des histoires. En règle générale, cette deuxième partie consacrée à l'étude des personnages qui appelle des tâches discursives non 
narratives est plus courte que la première, même si elle se révèle toujours d'un grand intérêt pour la compréhension fine de l'histoire.

\subsubsection{Le plan didactique} supports de séance. Les maitres racontent ou lisent les histoires non illustrées. Contrairement à une opinion assez répandue selon laquelle il serait assez difficile aux enfants de maternelle de maintenir leur attention à la seule écoute du texte lu, les élèves après une phase d'adaptation d'une ou deux séances prennent plaisir à ce nouveau mode de travail dont ils comprennent beaucoup plus facilement les enjeux cognitifs et linguistiques ainsi que des travaux menés ailleurs l'avaient déjà montré (Brigaudiot, 2012 ou Boiron, 2014). Une des conséquences de ce choix, certes prévisible, mais pourtant inattendue est qu'elle nous a obligés à porter une plus grande attention à la qualité et au contenu des textes que nous choisissions comme support de séance. Nous nous sommes donc orientés assez régulièrement vers des textes véritablement autonomes (Grossmann, $\left.1996 \mathrm{a}^{15}\right)$ : des contes traditionnels en particulier et en général des textes qui répondent aux critères définis par l'équipe Prog (Ducancel, 2006). Les histoires lues ou racontées font l'objet d'une préparation de la part des enseignants qui expliquent préalablement à la présentation de l'histoire les termes ou les expressions qu'ils jugent difficiles. C'est ainsi, pour une histoire, "Danger dans le potager $»^{16}$, qui a été reprise par de nombreux enseignants, que des expressions comme "pin centenaire» ou "sans la moindre inquiétude » ou encore les termes « remise » ou «intrépide » ont fait d'emblée ${ }^{17}$ l'objet d'une explication, parfois doublée lors de la lecture de reformulations paraphrastiques plus faciles à comprendre en contexte.

\subsubsection{Le plan pédagogique}

Sur le plan pédagogique, il est demandé aux enseignants d'adopter une posture en retrait favorable à la prise de parole de tous les élèves. Concrètement, la formation donne aux enseignants les moyens d'y accéder à travers la mise en œuvre du « dialogue pédagogique à évaluation différée » (Péroz, 2010) qui se caractérise d'abord par la réduction drastique de la vitesse des échanges et donc la réduction du nombre des questions (supra $\$ 131$ ), la répétition des questions ouvertes, ensuite l'interrogation de tous les élèves qui lèvent le doigt pour répondre à la question posée et enfin la possibilité laissé aux élèves de reprendre ce qui a déjà été dit pour construire leurs propres interventions. Sur l'ensemble du corpus, on note que cela ne débouche pratiquement jamais sur des répétitions strictes, même de la part des petits parleurs. Les reprises sont toujours des « reprises-reformulations ».

Nous allons maintenant présenter les résultats de la mise en œuvre de ce dispositif.

\section{Résultats}

On sait les difficultés de l'évaluation du langage oral à l'école (Garcia-Debanc, 2002). Quels indicateurs doit-on retenir? Comment prendre en compte les dimensions langagières et linguistiques de cet apprentissage qui à l'école maternelle est en partie aussi, l'apprentissage du langage lui-même?

Pratiques, 169-170 | 2016 
27 Pour pouvoir répondre à ces questions, l'un des buts du dispositif est de clarifier progressivement, tant pour l'enseignant que pour les élèves, les tâches discursives qui leur sont demandées. La segmentation de la séance en deux phases, l'une de restitution de l'histoire et l'autre de compréhension centrée sur les personnages contribue à cela. Le choix d'un texte non illustré comme support de séance appelle tout aussi clairement le développement d'un discours décontextualisé exigeant en matière de construction de la référence. De la même manière, la sélection de questions ouvertes qui reviennent de séance en séance permet aux élèves d'identifier la tâche discursive qu'on attend d'eux, d'autant plus nettement que l'enseignant ne les simplifie pas malgré les difficultés rencontrées. Mais l'enfant n'est pas seul. La posture en retrait de l'enseignant et le temps laissé pour réfléchir favorisent d'abord l'écoute entre élèves puis leur collaboration pour traiter des questions posées. Cela se traduit par l'allongement progressif de leurs interventions qui, dans ce cadre, est toujours le signe d'une meilleure maitrise du langage oral $^{18}$. Cet allongement résulte $d u$ travail énonciatif ${ }^{19}$ opéré par l'élève dans la construction même de son énoncé mais aussi du recours régulier à la reprise reformulation (auto ou hétéro) déjà observée comme un facteur de progrès vers le discours monologal tant pour les enfants de deux à trois ans (Salazar-Orvig, 2000) que pour les enfants plus âgés (François, 1993). Nous retrouvons ainsi les conditions de la "co-construction du langage oral » considéré par M. Grandaty (2002, p. 145) comme «l'objectif premier de ces classes » où « il s'agit moins pour l'enfant de se retrancher de l'interlocution que de s'aider de l'énoncé de l'interlocuteur, par reprise pour donner du "volume" à son tour de parole, volume senti intuitivement comme conduite discursive plus élaborée ».

Ces observations nous ont conduits à retenir l'allongement des interventions comme un indicateur central dans notre évaluation des progrès des élèves. Malgré son caractère inhabituel ${ }^{20}$, exception faite de travaux sur l'acquisition du langage avant trois ans et de quelques travaux sur lesquels nous reviendrons plus loin, cet indicateur présentait pour nous de multiples avantages. Il nous permettait un traitement statistique du corpus qui sans cela aurait été très difficile. Mais il donnait aussi un critère relativement objectif d'évaluation de la conduite des séances et donc un repère facilement mobilisable dans l'analyse conjointe des séances avec les enseignants du terrain ${ }^{21}$. Il nous donnait aussi un moyen de comparer nos résultats autrement que de manière qualitative avec ceux qui ont fait l'objet de mesures du même type dans la littérature existante. Enfin, il nous permettait, étant donné la taille et la relative homogénéité du corpus de donner des repères qui pourront eux-mêmes servir de points de comparaison pour les recherches à venir.

Une des hypothèses à l'origine de cette recherche est que, lors de la séance de langage, le travail énonciatif effectué par l'enfant constitue véritablement l'apprentissage du langage oral. La participation des élèves et l'allongement de leurs interventions sont donc les premiers objectifs poursuivis dans le cadre pédagogique mis en place. Pour apprécier les résultats obtenus sur ce plan nous les comparerons avec ceux des recherches qui poursuivent d'abord des objectifs langagiers, en l'occurrence les travaux d'A. Florin (1991) sur les groupes conversationnels dont l'objectif est clairement la participation des élèves. Cependant, même si les résultats sont bons sur ce plan, rien ne prouve à priori qu'il en est de même sur le plan linguistique. Nous aborderons donc ce point, en prenant deux critères plus classiques pour l'évaluation des compétences linguistiques des élèves: l'emploi des temps et le choix des connecteurs. 


\subsection{Participation des élèves et de l'enseignant au cours de la séance}

Les chiffres qui suivent portent sur l'ensemble des 78 séances enregistrées sur trois ans (2010-2013). Ils concernent d'abord la participation de l'enseignant et des élèves au cours des séances

\subsubsection{Présence verbale de l'enseignant au cours de la séance}

Dans le projet CLEA, la participation de l'enseignant est nettement réduite. Le nombre de ses interventions est inférieur d'un tiers à celui donné par A. Florin (1991) dans les groupes conversationnels pour un nombre d'élèves légèrement supérieur, 9 élèves en moyenne au lieu de 7,5 .

Tableau 2. Présence verbale de l'enseignant au cours des séances

\begin{tabular}{|l|l|l|}
\hline & $\begin{array}{l}\text { Nombre moyen d'élèves par } \\
\text { séance }\end{array}$ & $\begin{array}{l}\text { Interventions } \\
\text { l'enseignant }\end{array}$ \\
\hline $\begin{array}{l}\text { Groupes conversationnels (Florin, } \\
\text { 1991) } \\
(14 \text { séances MS / 14 séances GS) }\end{array}$ & 7,5 & $53 \%$ \\
\hline $\begin{array}{l}\text { CLEA (2010-2013) } \\
\text { (34 séances MS / } 44 \text { séances GS) }\end{array}$ & 9 & $36 \%$ \\
\hline
\end{tabular}

\subsubsection{Participation des élèves au cours des séances}

L'analyse des séances au fil des semestres comme les moments de formation et d'échanges avec les enseignants ont fait évoluer aussi bien les conceptions de l'équipe de recherche que le niveau de compétences professionnelles des enseignants.

Comme le montre le tableau ci-dessous, le nombre moyen d'interventions par élève augmente chaque année entre le premier et le deuxième semestre et il augmente entre la première et la deuxième année. On constate en effet, alors que les élèves ne sont pas souvent les mêmes d'une année sur l'autre, les enseignants qui, eux, ont pour la plupart une année d'expérience, obtiennent d'emblée de meilleurs résultats au début de la deuxième année.

Cette augmentation a pour corollaire un allongement relatif de la durée des séances qui témoigne du climat serein dans lequel elles se déroulent.

Tableau 3. Progression de la participation des élèves aux séances de langage

\begin{tabular}{|c|c|c|c|c|c|}
\hline & \multicolumn{2}{|c|}{$2010-2011$} & \multicolumn{2}{|c|}{ 2011-2012 } & 2012-2013 \\
\hline Semestres & 1 & 2 & 1 & 2 & 2 \\
\hline
\end{tabular}




\begin{tabular}{|l|l|l|l|l|l|}
\hline Nombre moyen d'interventions par élève par séance & 8,00 & 11,00 & 9,50 & 12,5 & 12,5 \\
\hline Nombre moyen d'interventions élèves par séance & 86 & 108 & 114 & 148 & 149 \\
\hline
\end{tabular}

\subsubsection{Longueur moyenne des interventions} échanges et donne une indication sur la vitesse des échanges. Par exemple, au cours de la séance sur «la bicyclette » en GS qui sert de support d'analyse à plusieurs études du recueil de l'INRP (Grandaty \& Turco, 2001), Oral dans la classe, la longueur moyenne des interventions élèves est de 5,5 mots, ce qui signifie que les phrases complexes articulant plusieurs propositions sont assez rares tandis que la vitesse des échanges est assez élevée. On peut supposer que la situation n'est guère différente dans les «groupes conversationnels» mis en place par A. Florin (1991) qui visent à des échanges individualisés et dont la longueur moyenne des interventions-élèves est de 5 mots. Elle note, elle-même (Florin, 1995, p. 126) que lors de séances de langage qu'elle a pu observer dans les classes, le nombre moyen de questions à la minute est de 6 et peut aller jusqu'à 10 avec certaines enseignantes.

Du point de vue linguistique, nous faisons l'hypothèse que la longueur moyenne des interventions est un indice du travail énonciatif accompli par l'élève. Mais jusqu'à quel point peut-on associer longueur de l'énoncé et maitrise syntaxique? Pour des adultes dont la syntaxe est assurée, la longueur moyenne des énoncés n'est pas significative de leur degré de maitrise de la langue. Mais, à l'école maternelle, la longueur moyenne des interventions lors d'une séance de langage reste un indice pertinent (Dewaele, 2000). Pour tous les auteurs qui travaillent sur l'acquisition du langage, la longueur est à cet âge un critère d'évaluation positif, qu'il s'agisse de psychologues comme A. Florin (1991, p. 74) ou de psycholinguistes spécialistes de l'évaluation du langage, comme J.-A. Rondal (1997), D. Bassano (2000, p. 160), B. Pierart (2005, p. 94) ou G. de Weck (2010, p. 155). Dès 1985, A. Florin et al. $(1985$, p. 200) l'explique dans des termes auxquels nous souscrivons pleinement :

En leçon de langage tout particulièrement la qualité des énoncés des enfants va de pair avec leur quantité; c'est lorsque les enfants prennent une part importante dans la conversation qu'ils construisent des phrases complètes et qu'ils introduisent des concepts nouveaux. Cela n'est en fait pas étonnant: si l'on ne prend la parole que pour compléter ou répéter ce que dit l'institutrice, des bribes de phrases suffisent; elles sont en quelque sorte insérées dans le discours de la maîtresse qui leur donne un sens. Mais il est évident que lorsque près de la moitié des énoncés sont émis par les enfants, ils seraient incompréhensibles s'ils ne dépassaient pas le niveau du syntagme. [...] Si l'on veut que les enfants parviennent à exprimer verbalement leurs pensées et leurs sentiments personnels, il faut évidemment les entraîner à produire des propositions et même des phrases à plusieurs propositions.

À cet égard, les résultats obtenus dans le cadre de notre projet, sont nettement meilleurs que ceux obtenus dans le cadre des groupes conversationnels, quel que soit l'âge des élèves ainsi que le montre le tableau ci-dessous dans lequel on a réparti en trois classes d'âge les élèves de grande et de moyenne section indépendamment de leur inscription dans ces classes. 
Tableau 4. Longueur moyenne des interventions en nombre de mots, par classe d'âge

\begin{tabular}{|l|l|l|l|}
\hline \multicolumn{2}{|l|}{$\begin{array}{l}\text { Nombre } \\
\text { d'élèves }\end{array}$} & \\
\hline 38 élèves & Âge 1 & de 3 ans 10 mois à 4 ans 7 mois & 9 \\
\hline 36 élèves & Âge 2 & de 4 ans 8 mois à 5 ans 6 mois & 12 \\
\hline 35 élèves & Âge 3 & de 5 ans 7 mois à 6 ans 4 mois & 16,5 \\
\hline
\end{tabular}

Comme le tableau le montre ci-dessus, le niveau des élèves n'est pas indépendant de leur maturité, ce qui est assez normal. Par contre, il montre aussi que les résultats obtenus sont nettement meilleurs que ceux qui sont obtenus dans le cadre de séances dans lesquels la présence de l'enseignant est prépondérante, même quand les objectifs affichés sont le développement des compétences langagières. C'est par exemple le cas, d'une séance de langage en grande section donnée en exemple aux enseignants dans un DVD ${ }^{22}$ $\mathrm{du}$ CRDP sur un thème bien connu des élèves dont la longueur moyenne des interventions ne dépasse pas 7, 5 mots. C'est aussi le cas des séances que nous avons pu observer dans les classes (Péroz, 2015a) quand elles sont menées sur le mode du dialogue pédagogique ordinaire pour reprendre l'expression d'É. Nonnon (1997).

Si l'on mesure le pourcentage d'énoncés comportant au moins un verbe conjugué, on constate que les résultats obtenus sont nettement meilleurs que ceux qui sont donnés par des élèves travaillant en groupe conversationnels (Florin, 1991).

Tableau 5. Pourcentage d'énoncés comportant au moins un verbe conjugué

\begin{tabular}{|l|l|l|}
\hline & MS & GS \\
\hline $\begin{array}{l}\text { Groupes conversationnels (Florin, 1991) } \\
\text { (14 séances MS / } 14 \text { séances GS) }\end{array}$ & $42 \%$ & $56,5 \%$ \\
\hline $\begin{array}{l}\text { CLEA (2010-2013) } \\
\text { (34 séances MS / } 44 \text { séances GS) }\end{array}$ & $74 \%$ & $80 \%$ \\
\hline
\end{tabular}

40 Mais dans quelle mesure la complexité des interventions est-elle liée à leur allongement ? Suivant en cela les travaux de M. Hickmann (2000) sur le développement de l'organisation discursive au-delà de trois ans, nous prendrons comme variables d'analyse, les temps verbaux et les connecteurs discursifs qui sont des marques essentielles de la cohérence du récit.

\subsection{Emploi des temps}

41 L'emploi des différents temps verbaux est un indice de maitrise de la langue et en particulier dans les conduites narratives. On sait (Rondal, 1998) que le passé composé est employé vers trois ans et qu'il précède l'apparition de l'imparfait vers cinq ans et celle 
plus tardive encore du conditionnel. Or la combinaison de ces trois temps, en particulier celle de l'imparfait et du passé composé est à la base de l'opposition des plans qui structurent le récit (Fayol, 2002). Nous faisons donc l'hypothèse que la présence de l'imparfait devrait s'accompagner d'un allongement des interventions, correspondant à la construction du deuxième plan dont il est la marque dans l'énoncé.

Pour le vérifier, nous avons dénombré dans le corpus ${ }^{23}$ les verbes employés aux temps du récit ${ }^{24}$ : le présent de narration, le passé composé puis les imparfaits et les conditionnels que nous avons regroupés dans les temps «en -ai » étant donné la proximité de leur fonctions sémantiques (Culioli, 1990 ; Lebaud, 1993).

Une même intervention peut mobiliser différents temps verbaux, il fallait donc définir un ordre pour indexer l'intervention à tel ou tel temps. À priori, les interventions les plus simples sont au présent de narration, ce sont d'ailleurs les enfants les plus jeunes qui le mobilisent le plus souvent mais s'il y a un temps du passé en particulier un passé composé, l'intervention est indexée à ce temps, enfin s'il se trouve un temps en -ai c'est ce dernier qui est pris en compte pour indexer l'intervention. La présence de l'imparfait suppose en général la présence concomitante d'un des temps du premier plan ${ }^{25}$, passé composé ou présent de narration. C'est donc avec l'imparfait que le système se développe vraiment. Les temps du premier plan comme le présent de narration ou le passé composé peuvent dès lors apparaitre sur le fond des commentaires, des descriptions ou des explications données à l'imparfait ; ainsi dans les exemples suivants.

4,11 48 Cyrielle Pierre / Pierre / il était, il était dans l'arrosoir plein de glace / plein d'eau froide / et quand il est allé / eh ben / il a atchoumé / et monsieur Grégory s'est retourné

5,00 35 Soheib il saute dans l'arrosoir et c'était vraiment pas une bonne idée / parce que c'était de l'eau glacée / il a fait a / atch / atchoum / et monsieur Grégory / il s'est retourné et après il a couru si vite

Dans le système à trois temps que nous étudions ici, l'imparfait est le temps dont la présence augmente le plus nettement au fur et à mesure de la scolarité, tandis que le nombre d'interventions au présent de narration diminue et que stagne ou diminue celui des interventions au passé composé.

Tableau 6. Évolution de la présence relative des temps en fonction de l'âge des élèves

\begin{tabular}{|l|l|l|l|l|}
\hline & Âge 1 & Âge 2 & Âge 3 & Total \\
\hline Temps en -ai & $19 \%$ & $27 \%$ & $35 \%$ & $28,5 \%$ \\
\hline Passé composé & $26 \%$ & $24 \%$ & $25,5 \%$ & $25 \%$ \\
\hline Présent de narration & $34,5 \%$ & $25 \%$ & $14,5 \%$ & $22,5 \%$ \\
\hline Autres temps & $21 \%$ & $24 \%$ & $25 \%$ & $24 \%$ \\
\hline Nombre d'interventions & 773 & 3692 & 2069 & 6534 \\
\hline
\end{tabular}

Mais le point le plus intéressant est sans doute que les interventions qui comportent un imparfait sont d'emblée nettement plus longues que les autres tandis que la progression de 
la longueur des interventions au passé composé semble plus dépendante de la maturité des élèves.

Tableau 7. Évolution de la longueur moyenne des interventions aux temps en -ai et au PC

\begin{tabular}{|l|l|l|l|}
\hline & Âge 1 & Âge 2 & Âge 3 \\
\hline Temps en -ai & 18,7 & 19,3 & 22,5 \\
\hline PC & 11,8 & 13,2 & 15,2 \\
\hline
\end{tabular}

Il est sans doute assez vain de se prononcer sur l'antériorité de l'un ou l'autre des deux paramètres que sont ici la longueur des interventions et la présence d'un temps en -ai. Par contre, il est clair qu'en deçà d'un certain seuil, en deçà d'une certaine longueur que l'on peut facilement estimer à une douzaine de mots, on ne permet pas aux élèves l'apprentissage des emplois de ces temps, ce qui renvoie directement au choix du modèle d'apprentissage du langage oral que l'on mobilise.

Mais ces interventions correspondent-elles véritablement à ces énoncés complexes que les programmes attendent de nos élèves à la fin du cycle 1 ? Un des indicateurs dont on dispose est la présence et la nature des connecteurs dans l'intervention.

\subsection{Emploi des connecteurs}

On a quelques connaissances dans le domaine, on sait par exemple que les connecteurs temporels et en particulier ceux qui marquent une succession comme et (interpropositionnel), et après, quand, après, et puis vont être les premiers utilisés. Ces cinq connecteurs sont nettement plus fréquents que les autres dans notre corpus comme le montre le tableau ci-dessous. On notera au passage, la présence de l'archi-connecteur et qui est non seulement le plus fréquent et de loin mais qui constitue aussi nombre des locutions temporelles de ce tableau.

Tableau 8. Fréquence des connecteurs temporels par ordre décroissant

\begin{tabular}{|l|l|l|l|l|l|}
\hline et & $\mathbf{1 9 7 7}$ & et puis après & 39 & et ensuite & 16 \\
\hline et après & $\mathbf{5 8 5}$ & et quand & 35 & et après quand & 14 \\
\hline quand & $\mathbf{3 8 9}$ & après que & 31 & dès que & 12 \\
\hline après & $\mathbf{3 2 7}$ & puis & 23 & et maintenant & 11 \\
\hline et puis & $\mathbf{1 4 4}$ & au début & 22 & avant que & 11 \\
\hline
\end{tabular}

\subsubsection{Les connecteurs temporels de base}

51 La première observation est que la présence de ces connecteurs temporels de base ne diminue pas, au contraire, avec l'âge des élèves ${ }^{26}$, comme le montre le tableau suivant qui 
donne, pour l'ensemble du corpus, le pourcentage d'interventions comportant au moins un de ces connecteurs selon l'âge des élèves.

Tableau 9. Pourcentage des interventions comportant au moins un connecteur temporel de base

\begin{tabular}{|l|l|l|}
\hline Âge 1 & Âge 2 & Âge 3 \\
\hline $44,50 \%$ & $45 \%$ & $70 \%$ \\
\hline
\end{tabular}

Ainsi comme l'avait déjà noté J.-M. Colleta (2004), l'allongement de l'intervention avant 6 ans se traduit selon une macro syntaxe additive par une augmentation équivalente du nombre des propositions et des connecteurs, en particulier temporels. En voici un exemple au passé composé, à propos du " Joueur de flûte de Hamelin » :

4,10 3 Jules et après le maire il a fait venir les souris il a / il les a mis(es) dans l'eau il les a / et le maire il a / il a décidé de donner cent pièces et après il a joué de la musique il / la montagne s'est refermée et tous les enfants sont venus

La seconde observation est que comme pour la longueur des interventions, la densité des connecteurs temporels dans les interventions comportant un imparfait est d'emblée très importante et semble nettement moins liée à un paramètre développemental que ne l'est l'évolution correspondante au passé composé ou aux autres temps.

Tableau 10. Pourcentage des interventions comportant au moins un connecteur temporel de base en fonction du temps indexant l'intervention et l'âge de l'élève

\begin{tabular}{|l|l|l|l|}
\hline & Âge 1 & Âge 2 & Âge 3 \\
\hline Temps en -ai & $92,50 \%$ & $75,50 \%$ & $93,00 \%$ \\
\hline Passé composé & $38,50 \%$ & $45,00 \%$ & $64,00 \%$ \\
\hline Présent de narration & $31,00 \%$ & $25,00 \%$ & $31,00 \%$ \\
\hline Autres temps & $31,00 \%$ & $29,50 \%$ & $63,00 \%$ \\
\hline
\end{tabular}

55 Dans un exemple comme le suivant, d'un élève de 4 ans et 4 mois, l'imparfait associé au passé composé permet de développer au deuxième plan les perceptions inquiétantes du personnage, l'ensemble étant clairement scandé par le connecteur temporel et après.

4,04 12 Fiona et après / le petit chaperon vert ${ }^{27}$ / et après / il a couru / et après / dans le noir avec ses yeux brillants / dans le noir il a vu des yeux qui couraient / ils couraient doucement sans faire de bruit

\subsubsection{Les connecteurs logiques}

Nous avons mesuré de la même manière la présence dans les interventions des connecteurs logiques. Il s'agit par rang décroissant de fréquence de : parce que, si, comme, alors, comme ça, sinon, pour que, donc, et en fait. Leur densité est moitié moindre que celle des connecteurs temporels ( $27 \%$ au lieu de $53 \%$ ) sur l'ensemble des interventions. 
Comme les connecteurs temporels, les connecteurs logiques ${ }^{28}$ apparaissent le plus souvent dans des interventions qui comportent au moins un imparfait. Mais leur évolution est très forte au fil du développement, comme si, dans un système verbal complexe comme c'est le cas lorsqu'il y a un temps en -ai, les possibilités cognitives et expressives de l'élève se traduisaient plus nettement par ce type de marqueur.

Tableau 11. Évolution de la présence de connecteurs logiques selon l'âge de l'élève et selon les temps employés dans les interventions

\begin{tabular}{|l|l|l|l|}
\hline & Âge 1 & Âge 2 & Âge 3 \\
\hline Temps en -ai & $25,52 \%$ & $31,59 \%$ & $55,43 \%$ \\
\hline Passé composé & $13,07 \%$ & $16,35 \%$ & $27,99 \%$ \\
\hline Présent de narration & $17,16 \%$ & $18,72 \%$ & $34,78 \%$ \\
\hline Autres temps & $11,18 \%$ & $21,24 \%$ & $29,09 \%$ \\
\hline
\end{tabular}
l'expression est rendue possible par la présence de l'imparfait comme il rend possible l'expression des hypothèses.

4,09 178 Isaac il faut pas y aller dans le potager ${ }^{29}$ parce que il y a monsieur Grégory et il surveille tout et-euh-euh déjà qu'il avait vu Pierre alors euh Pierre il a entendu un bruit étrange et euh du coup il a il a pris sa course folle

4,11 97 Cyrielle on écouterait la maman si on était dans la famille de Pierre et on n'irait pas dans le jardin de monsieur Grégory parce que il a plein de dangers / et si on y va / il va nous transformer en pâté.

6,00 136 Théodore il (n')y en a qu'un qui a été dans le potager c'est Pierre / parce que la maman elle avait dit / vous pouvez aller dans le pré / donc donc ils sont pas... donc ils ont... donc Capucine Romarin et Neige qui sont les plus obéissants ils ont été donc dans le pré que la maman avait dit d'aller / ou (bien) ils seraient restés dans la maison / mais ils ont pas tous désobéi... /// que un (a désobéi)

1 Ces connecteurs logiques vont apparaitre en particulier dans la deuxième partie de la séance qui porte sur les personnages, leurs états mentaux ou leurs motivations dont 4,10 153 Tristan j'ai entendu elle avait éclatée la moufle ${ }^{30}$ parce que si la mouche elle rentrait eh ben la moufle elle elle éclatait

5,00 197 Jules ben en fait il voulait le transformer en pâté mais il a pas réussi hein

58 Shanna non / parce que le renard il voulait pas $1 \mathrm{e}^{31}$ manger tout de suite / il voulait le laisser refroidir

6,01 174 Alicia si j'étais monsieur Grégory je laisserais les lapins tranquilles / je les laisserais dans mon jardin cueillir tous les fruits

5,07 38 Thomas et Cochonnet ${ }^{32}$ il avait marre / c'était les parents qui l'énervaient le plus Loupinette 


\section{Conclusion}

L'analyse des séances de langage réalisées pendant les trois ans du projet CLEA montre que le choix d'un modèle d'apprentissage qui favorise d'abord la prise de parole des élèves dans un contexte exigeant tant pour le nombre d'élèves présents que pour le support retenu, un texte non illustré lu par l'enseignant, donne des résultats supérieurs à ceux que l'on peut obtenir dans les séances organisées sur un modèle dialogal adultocentré. Le dispositif proposé aux enseignants permet d'augmenter la participation des élèves tant pour le nombre que pour la longueur de leurs interventions. Il permet aux élèves de s'engager dans un véritable travail énonciatif qui malgré ses difficultés et ses erreurs est à la base de l'apprentissage du langage oral. Cela est confirmé par l'augmentation progressive du nombre de connecteurs temporels qui viennent scander une syntaxe additive, au passé composé et autres temps tandis que la progression rapide du nombre des connecteurs logiques semble associée plus directement au développement des compétences cognitives et discursives des élèves. L'ensemble des résultats montre que les temps en -ai jouent un rôle essentiel dans le développement du langage. Quel soit l'âge des élèves, leur présence dans l'intervention correspond d'une part à un allongement d'un tiers de l'intervention par rapport aux autres temps, d'autre part à la présence systématique de connecteurs temporels et enfin s'accompagne de la progression la plus forte des connecteurs logiques.

Les différents critères dont nous venons d'évaluer la présence dans les interventions des élèves ne seraient pas à ce niveau sans une réelle appropriation des textes dont les quelques exemples cités ne donnent qu'un aperçu. Il resterait à le montrer précisément ${ }^{33}$, mais le lecteur nous en sera gré, nous nous en tiendrons là.

\section{BIBLIOGRAPHIE}

ASTOLFI, J.-P. (1993). « Trois paradigmes pour les recherches en didactique ». Revue française de pédagogie 103, p. 5-18.

BASSANO, D. (2000). « La constitution du lexique : le "développement du lexique précoce" ». In : Kail, M. \& Fayol, M., L'acquisition du langage, t. 1, Le langage en émergence De la naissance à trois ans. Paris : Presses universitaires de France.

BAUTIER, É. (dir.) (2008). Apprendre à l'école. Apprendre l'école. Des risques de construction d'inégalités dès la maternelle. Lyon : Chronique sociale.

BAUTIER, É. \& GOIGOUX, R. (2004). « Difficultés d'apprentissage, processus de secondarisation et pratiques enseignantes : une hypothèse relationnelle ». Revue française de pédagogie 148, p. 89-100. BISHOP, M.-F. \& JOOLE, P. (2012). « Et si l'on parlait des récits... ». Le Français aujourd'hui 4, 179, p. 3-8. 
BOIRON, V. (2014). « Raconter et lire des récits de fiction : effets comparés sur la compréhension d'élèves de maternelle ». Repères 50, p 83-104.

BOIRON, V. \& REBIÈRE, M. (2009). « Quels albums pour la petite section ? Propositions de critères de choix ». Diptyque 17, p. 11-26.

BRAUN-LAMESCH, M. M. (1972). « Le rôle de l'imitation dans l'acquisition du langage ». Enfance 25, 5, p. 397-417.

BRIGAUDIOT, M. (dir.) (2000), Apprentissages progressifs de l'écrit à l'école maternelle, Paris : INRP/ Hachette.

- (2012). «Sciences du langage ». Le Français aujourd'hui 4, 179, p. 129-138.

BRUNOT, F. \& BRUNEAU, C. (1949). Précis de grammaire historique de la langue française. Paris: Masson.

CÈBE, S. \& GOIGOUX, R. (2012). « Comprendre et raconter : de l'inventaire des compétences aux pratiques d'enseignement ». Le Français aujourd'hui 4, 179, p. 21-36.

COLLETA, J.-M. (2004). Le développement de la parole chez l'enfant âgé de 6 à 11 ans. Corps, langage et cognition. Wavre (Belgique) : Mardaga.

COLLINOT, A. \& MAZIÈRE, F. (1999). Le français à l'école Un enjeu historique et politique. Paris : Hatier. Culioli, A. (1990). «Valeurs modales et opérations énonciatives ». In : Culioli, A. Pour une linguistique de l'énonciation, t. 1, Opérations et représentations. Paris : Ophrys, p. 135-156. DANNEQUIN, C. (1976). Les enfants bâillonnés. Paris : Sudel.

DEWAELE, J.-M. (2000). « Saisir l'insaisissable ? Les mesures de longueur d'énoncés en linguistique appliquée ». International Review in Applied Linguistics in Language Teaching 38, p. 17-33.

DUCANCEL, G. (coord.) (2006). Sens et code au cycle 2 Apprentissages progressifs de l'écrit au cycle 2, PROG. Paris : INRP/Hachette éducation.

FAYOL, M. (1994). Le récit et sa construction. Une approche de psychologie cognitive. Genève : Delachaux \& Niestlé éds.

- (dir.) (2002). Production du langage. Paris : Hermès Science publications.

FLORIN, A. (1991). Pratiques du langage à l'école maternelle et prédiction de la réussite scolaire. Paris : Presses universitaires de France.

- (1995). Parler ensemble en maternelle. La maîtrise de l'oral, l'initiation à l'écrit. Paris : Ellipses.

FLORIN, A., BRAUN-LAMESCH, M. M. \& BRAMAUd du BOUCHERON, G. (1985). Le langage à l'école maternelle. Bruxelles : Mardaga.

FRANÇOIS, F. (1993). Pratiques de l'oral. Dialogue, jeux et variations des figures du sens. Paris : Nathan.

GARCIA-DEBANC, C., (2002). « Enseigner l'oral ?». Repères 24-25, p. 3-21.

GARCIA-DEBANC, C. \& PLANE, S. (coord.) (2004). Comment enseigner l'oral à l'école primaire ? Paris : INRP/Hatier éd.

GIASSON, J. (1996). La compréhension en lecture. Paris : De Boeck.

GRANDATY, M. (2002). « Évaluation des apprentissages oraux au cycle 1 ». Repères 24-24, p. 135-150.

GRANDATY, M. \& TURCO, G. (coord.) (2001). L'oral dans la classe. Discours, métadiscours, interactions verbales et construction de savoirs à l'école primaire. Paris : INRP éd. 
Grossmann, F. (1996a). Enfances de la lecture. Berne/Paris : Peter Lang.

- (1996b). «Que devient la littérature enfantine lorsqu'on la lit aux enfants d'école maternelle

?»Repères 13, pp. 85-101.

HALTÉ, J-F. (1992). La didactique du français. Paris : Presses universitaires de France.

JAUBERT, M. \& REBIÈRE, M. (2011). « Le genre scolaire "lecture découverte" d'un texte narratif au Cours Préparatoire : un outil pour la formation ». In : Goigoux, R. \& Pollet, M.-C. (dirs), Didactiques de la lecture, de la maternelle à l'université. Namur : Presses universitaires de Namur, p. 53-77.

KARMILOFF, K. \& KARMILOFF-SMITH, A. (2003). Comment les enfants entrent dans le langage. Paris : Retz.

HICKMANN, M. (2000). « Le développement de l'organisation discursive ». In : Kail, M. \& Fayol, M., L'acquisition du langage, t. 1, Le langage en émergence De la naissance à trois ans. Paris : Presses universitaires de France, p. 83-116.

LAHIRE, B. (1993). Culture écrite et inégalités scolaires. Lyon : Presses universitaires de Lyon.

LEBAUD, D. (1993). «L'imparfait : indétermination aspectuo-temporelle et changement de

repère ». Le gré des Langues 5, p. 160-177.

MAKDISSI, H., BOISCLAIR, A. \& FORTIER, C. (2007). « Description du développement de l'utilisation des connecteurs chez les enfants du préscolaire en fonction de la structuration de leur rappel de récit ». Recherche Qualitative 27, 1, p. 99-127.

Ministère de l'Éducation nationale (MEN) (1999). La place de l'oral dans les enseignements à l'école primaire. Rapport n 99-024 de l'Inspection générale de l'Éducation nationale (M. Safra, J. Hébrard \& S. Thévenet rapporteurs).

- (2011). L'école maternelle. Rapport n 2011-108 de l'Inspection générale de l'Éducation nationale (V. Bouysse, P. Claus et Christine Szymankiewicz rapporteurs).

NONNON, É. (1997). «Quels outils se donner pour lire la dynamique des interactions et le travail sur les contenus de discours?». Enjeux 39-40, p. 12-49.

- (1999). « Note de synthèse : L'enseignement de l'oral et les interactions verbales en classe : champs de référence et problématiques ». Revue française de pédagogie 129, p. 87-131.

- (2002), « Des situations problèmes pour la verbalisation : difficultés à dire et travail de l'énonciation ». Repères 24-25, p. 23-58.

PÉROZ, P. (2010). Apprentissage du langage oral à l'école maternelle. Pour une pédagogie de l'écoute. Nancy : Scéren/CRDP de Lorraine.

- (2014). " parce que son papa, madame Grégory a fait un civet, un civet avec le papa ». Sur les procédures d'apprentissage du lexique : reprise et reformulation en langage oral à l'école maternelle. Congrès Mondial de Linguistique Française, 19-23 juillet. En ligne : http://www.shsconferences.org/articles/shsconf/pdf/2014/05/shsconf_cmlf14_01221.pdf.

- (2015a). « Apprentissage du langage oral à l'école maternelle. Quel modèle ? ». In : Carton, F. et al., Cultures de recherche en linguistique appliquée. Paris : Riveneuve, p. 189-206.

- (2015b). « Apprendre ensemble à parler et à raconter à l'école maternelle ». In : PNF. (DGESCO). Le rendez-vous des lettres « Les métamorphoses du récit à l'heure du numérique : récit et valeurs, valeurs de la fiction », 23 et 24 novembre 2015. Paris : BNF. F. Mitterand. En ligne : http:// eduscol.education.fr/pnf-lettres/spip.php?article319.

PERRENOUd, P. (1991). « Bouche cousue ou langue bien pendue ? L'école entre deux pédagogies de l'oral ». In : Wirthner, M. et al., Parole étouffée, parole libérée. Fondements et limites d'une pédagogie de l'oral. Neuchâtel : Delachaux et Niestlé.

PIERART, B. (2005). Le langage de l'enfant : comment l'évaluer ?, Bruxelles : De Boeck. 
QUET, F. (2009). « Qui résiste aux albums résistants? » Diptyque 17, p. 97-114.

REUTER, Y. (1988). « L'importance du personnage ». Pratiques 60, p. 3-22.

RONDAL, J-A. (1997). L'évaluation du langage. Liège : Mardaga.

WECK, G. de (2010), « Interactions adulte-enfant et troubles du développement du langage : bilan

des recherches et questions ouvertes ». In : Bernicot, J. et al. (éds), Interactions verbales et

acquisition du langage. Paris : L'Harmattan, p. 151-170.

WinNYKAMEN, F. (1990). Apprendre en imitant. Paris : Presses universitaires de France.

\section{NOTES}

1. Dans le rapport le plus récent $(2011: 124)$, le sous-titre introductif à la partie consacrée à notre sujet est assez clair: «La pédagogie de l'oral : toujours autant de faiblesses et les mêmes difficultés ».

2. Citons pour mémoire Les enfants bâillonnés de C. Dannequin (1976) ou plus récemment Parler ensemble en maternelle d'A. Florin (1995).

3. Le groupe CLEA ou «Comment Les Enfants Apprennent à parler à l'école maternelle » est une émanation du groupe Praxitexte qui lui-même appartient au Centre de recherche et d'étude sur les médiations (EA 3476) de l'Université de Lorraine.

4. Cette représentation est parfois confirmée par les textes de référence comme les Programmes 2008 (p. 12) qui font de l'enseignant un maitre à parler qui de manière assez peu réaliste et certainement anxiogène "veille à offrir constamment à ses jeunes élèves un langage oral dont toute approximation est bannie ; dont le discours ne souffre aucune approximation ».

5. Les documents d'accompagnement des Programmes 2008 confirment cette surreprésentation de la parole magistrale dans les échanges, véritable obstacle à la prise de parole des élèves : «Trop rare dans les séances de langage saturées par la parole magistrale, le silence du maître permet aux enfants de "se lancer" et d'aller jusqu'au bout de leur propos en enchaînant plusieurs phrases. L'enregistrement de séances de langage montre que ces occasions sont trop rarement offertes aux enfants ». (Langage à l'école maternelle, 2011, Scéren/CRDP).

6. M. M. Braun-Lamesch (1972) discute le caractère opératoire de l'imitation selon les niveaux de structuration de l'énoncé mais ne remet pas en cause son rôle dans l'apprentissage du langage oral en général, en particulier sur le plan lexical et sémantique.

7. Cette prolongation du projet initial a été rendue possible grâce au soutien du CARDIE de l'académie.

8. Le recours à un tableur a permis de traiter, au moins pour certains critères, la masse des données dont nous disposions. Le décompte des mots en particulier a pu être fait grâce à une formule de calcul qu'on ne pourrait utiliser dans un traitement de texte. Pour éviter de dénombrer des répétitions dues aux hésitations assez normales à l'oral, elles n'ont pas été comptées au-delà de la première.

9. Ce temps est de 18 heures par an (Circulaire $n^{\circ} 2011-042$ du 22 mars 2011).

10. Au bout d'un an de pratique, les enseignants ont adopté avec succès la posture en retrait qui leur était proposée. Mais ensuite, la question s'est posée de savoir quand et comment il faut sortir du retrait pour soutenir un élève, fournir un mot sur lequel il bute, ou encore quand il faut valider telle ou telle proposition pour aller plus avant. On entre alors dans cette « finesse du guidage pédagogique» dont parle E. Nonnon (2014), que nous considérons comme une compétence professionnelle experte en cela qu'elle se caractérise par des décisions qui articulent, en situation, des connaissances théoriques assurées et une connaissance effective des 
procédures d'apprentissage des élèves en cause. Le concept de «format de séance » ici évoqué est rapidement présenté dans P. Péroz (2015a) et infra au $§ 1.3$.

11. Les enseignants avaient le choix des supports lors de la première année : album ou texte non illustré ou encore histoire racontée sans support. Par la suite, tous les enseignants racontent l'histoire ou lisent un texte non illustré ; ils n'utilisent pas d'album comme support de séance.

12. C'est-à-dire tous les programmes depuis 1995 et leurs documents d'accompagnement en 2006, 2011 ou 2015.

13. On retrouve au cours de ces séances les deux paradigmes fondateurs de la discipline français (Collinot \& Mazière, 1999) : « celui de la composition (production) rhétorique au moyen de transformation d'énoncés " avec le rappel plus ou moins collectif du récit « d'où la nécessité du beau texte et de tout ce qui va avec », et le paradigme herméneutique, de la médiation, de l'interprétation : la phase de compréhension et tout ce qui va avec le questionnement ». Ce type de séance fait partie des «dispositifs» que des auteurs comme C. Garcia-Debanc et S. Plane (2004 : 226) proposent pour travailler l'oral.

14. Le choix de la chronologie comme un critère de la maitrise de base des conduites narratives, que l'on retrouve à peu près toujours dans les objectifs poursuivis lors des séances de langage sur des supports narratifs, et ce dès les premières séances sur un même support, repose sur une confusion classique entre une compétence experte, telle qu'elle a pu être scientifiquement décrite (Fayol, 1994), et les compétences à construire lors de l'apprentissage pour atteindre ce niveau.

15. L'auteur écrit, page 91 : «Si la forme album, par sa complexité même, est un outil culturel extrêmement intéressant, apprivoisant des langages différents, elle ne prépare guère, en raison de sa spécificité, à la lecture du texte écrit autonome. »

16. Le texte support est une réécriture de Danger dans le potager. Une aventure de Pierre Lapin de B. Potter adapté en français par S. Monnier-Murariu, illustré par L. Mc Cue, éditions Lipokili, 2005. Elle met en scène un lapin, Pierre, aux prises avec un jardinier M. Grégory auquel il a chapardé quelques légumes.

17. Les erreurs récurrentes mais qui signalaient par leur répétition un travail élaboratif en cours ont donné lieu à des séances « décrochées » qui avaient pour objet un enseignement explicite de la structure en cause, enseignement que les élèves pouvaient réinvestir de manière fonctionnelle lors des séances de langage suivantes. Les constructions verbales par exemple, comme se détacher de/se prendre ont pu faire l'objet de ces séances. Les Programmes 2015 ont confirmé l'intérêt de ces «moments structurés» d'apprentissage du langage oral. (Eduscol, Ressources maternelle, Partie I, L'oral, Cadrage général), distincts des séances de langage proprement dites.

18. À cet âge, et dans ce cadre. Lors de la séance de langage, les élèves ne sont pas dans une situation informelle mais dans une situation dans laquelle la prise de parole, savante par définition, demande de la part du locuteur un engagement personnel et cognitif d'autant plus réel que le discours s'allonge, devenant progressivement plus exigeant tant sur le plan de la gestion des informations que sur le plan linguistique.

19. Travail énonciatif, c'est-à-dire, lors de la séance de langage : «la forme de travail la moins lisible mais la plus constante, qui tient à l'activité énonciative elle-même, et que potentialisent les activités de verbalisation attachées aux situations d'apprentissage de l'école. Cette dimension, la plupart du temps invisible, se manifeste ordinairement quand la parole rencontre la résistance du langage, soit dans son ajustement aux choses à dire (dans des situations où on ne dispose pas de solutions linguistiques précodées), soit quand on peine à établir l'inter-compréhension avec autrui : ce sont, en fait, les difficultés à dire qui en font prendre conscience.» (Nonnon, 2002, p. 25).

20. À ce jour, la grande majorité des études sur l'apprentissage du langage oral à l'école maternelle est de type qualitatif. Voir sur ce point la recension des travaux dans la synthèse d'E. Nonnon (1999), toujours d'actualité. 
21. Il nous a permis par exemple, de montrer aux enseignants à quel point la phase de restitution est importante pour l'apprentissage du langage dans la mesure où elle permet aux élèves de s'engager dans le discours monologal dont la maitrise progressive est essentielle dans la scolarité mais montrer aussi que la deuxième phase de la séance donnait bien lieu à des conduites discursives différentes sinon plus difficiles et qui donnaient lieu à des interventions plus courtes.

22. La séance conduite par un EMF regroupe 13 élèves, elle vient clore une séquence didactique sur le thème des « Indiens » d'Amérique. CRDP, DVD Apprendre à parler, 2010.

23. On sait que les mesures moyennes sont sensibles aux extrêmes, pour éviter ces distorsions nous n'avons donc pas pris en compte 1807 interventions : les plus longues et les plus courtes, soit $22 \%$ du corpus qui compte dès lors 6534 interventions élèves. Nous avons en particulier écarté les interventions qui n'ont pas abouti, l'élève ne réussissant pas vraiment à parler, les interventions composées de groupes nominaux sans verbe conjugué dont la longueur moyenne de 3 mots ne varie pas, ce qui est somme toute logique, et à l'autre extrême les interventions qui comptent plus de 100 mots. Nous avons donc écarté les 60 interventions de plus de 100 mots, les 203 interventions qui n'ont pas abouti, et les 1607 interventions ne comportant pas de verbes conjugués et donc formée sur un groupe nominal ou un infinitif.

24. Le passé simple n'apparait que dans $2 \%$ des interventions (soit 136 interventions sur le total de 6534 ). C'est la raison pour laquelle il n'est pas décompté ici dans les temps du récit mais regroupé avec les «autres temps » utilisé par les élèves : présent d'actualité, futur, présent gnomique.

25. Pour F. Brunot et C. Bruneau (1949), l'imparfait est un temps « dépendant ».

26. Par contre, il y a des chances pour que l'extension sémantique de ces « opérateurs discursifs » diminue au profit d'emplois proches des emplois normés (Makdissi et al., 2007).

27. Le texte support de séance est le «Le petit chaperon vert ", extrait de Le grand méchant livre du loup, Bayard jeunesse, 2003.

28. Comme on a déjà pu le constater (Makdissi et al., 2007) pour des connecteurs discursifs ayant des fonctions structurantes dans le récit, ces connecteurs logiques sont plus sensibles à l'âge des élèves. La courbe d'augmentation du premier au troisième âge est très nette de 29,38 à $50 \%$.

29. Voir note 16.

30. On a reconnu le conte russe, La moufle.

31. Il s'agit du Petit bonhomme de pain d'épice que le renard ne semble pas vouloir immédiatement. Shana, pariant sur un rapprochement avec Roule Galette suppose qu'il préfère le laisser refroidir.

32. Le texte support de séance est extrait de Un cochon chez les loups de F. Stehr, Paris, L'école des loisirs, 2004.

33. Un travail sur les modalités d'apprentissage du lexique s'appuyant sur l'analyse de ce même corpus a fait l'objet d'une communication (Péroz, 2014).

\section{RÉSUMÉS}

L'apprentissage du langage oral à l'école maternelle fait l'objet d'observations convergentes de la part des chercheurs comme de celle des personnels d'inspection $(1999,2011)$ depuis de nombreuses années. Tous pointent la contradiction entre les objectifs affichés aux séances dites de langage et une parole magistrale qui occupe plus de la moitié de l'espace conversationnel. Le modèle d'apprentissage de ces séances, le modèle « dialogal adulto-centré » qui repose sur l'idée 
que comme dans la famille, «l'enfant apprend à parler avec l'adulte » a pour conséquence de privilégier l'individualisation des échanges mais aussi l'accélération du dialogue maitre - élèves. Ce dispositif favorise par définition les échanges rapides et exclut les élèves les moins performants ou les moins cultivés. Trente pour cent des élèves ne prennent pas la parole et la longueur moyenne des interventions des autres élèves est de 6 à 8 mots (Florin, 1991), bien loin de ce qui serait nécessaire pour construire les « phrases complexes » ou acquérir les compétences discursives « raconter, décrire, expliquer etc.» attendues en grande section par les Programmes (2015) pour l'école maternelle. La recherche CLEA (2010-2013) dont les résultats sont présentés ici, montre que la mise en œuvre d'un modèle d'apprentissage qui se donne explicitement comme objectif le développement des compétences langagières est possible et plus efficient, quand l'ensemble des paramètres de l'acte d'enseignement est pris en compte (régulation des échanges, choix des supports, apprentissages, format de séance). L'auteur présente d'abord les éléments constitutifs du modèle "polylogal» puis la méthodologie de recherche qui a conjugué la formation des maitres, le recueil et l'analyse des données, au total 78 séances en grande et moyenne section de maternelle, enregistrées et transcrites, soit un fichier numérique de quelques 140000 mots. La numérisation du corpus permet de montrer que statistiquement, les résultats en termes de participation et de longueur d'intervention sont nettement meilleurs que ceux obtenus dans les groupes qui tout en poursuivant les mêmes objectifs mettent en œuvre le modèle d'apprentissage habituel (Florin, 1991). L'allongement des interventions se traduit par ailleurs, sur le plan linguistique par une meilleure maitrise de l'emploi des temps du récit et des connecteurs temporels.

Learning oral language to preschool been converging observations by researchers as the personal inspection $(1999,2011)$ for many years. All point to the contradiction between the stated objectives of the sessions say language and masterful speech which occupies more than half of the conversational space. The learning from these sessions model, the "dialogical adultocentered" based on the idea that as in the family, "the child learns to speak with the adult" has the effect of favoring the individualisation of trade, but also accelerating the master-students dialogue. This device by definition promotes the rapid exchange and excludes the least efficient and least educated students. Thirty percent of pupils do not speak and the average length of interventions of others is 6 to 8 words (Florin, 1991), far from what is needed to build the "complex sentence" or acquire the discursive skills "narrate, describe, explain, etc." expected by the programs (2015) for kindergarten. CLEA research (2010-2013), whose results are presented here shows that the implementation of a learning model that explicitly gives objective the development of language skills is possible when all the parameters of educational act is taken into account (trade regulation, choice of media, learning, meeting format). The author first introduces the elements of the model "polylogal" and the research methodology that combined the training of teachers, the collection and analysis of data, a total of 78 sessions in large and medium class, recorded and transcribed, or a digital file of some 140,000 words. The digitization of the corpus has shown that statistically, the results in terms of participation and intervention length are significantly better than those obtained in groups while pursuing the same objectives implement the usual learning model (Florin, 1991). Longer interventions are reflected also linguistically through better control of the use of narrative time and time slots.

\section{INDEX}

Mots-clés : apprentissage du langage oral, école maternelle, modèle d'apprentissage, analyse de corpus

Keywords : learning spoken language, kindergarten, learning model, corpus analysis 
AUTEUR

PIERRE PÉROZ 